University of Wollongong

Research Online

Faculty of Engineering and Information

Faculty of Engineering and Information

Sciences - Papers: Part B

Sciences

2019

Theoretical study on positron scattering by benzene over a broad energy

range

Alessandra Souza Barbosa

Federal University of Parana

Francisco Blanco

Complutense University of Madrid

Gustavo Garcia

Superior Council of Scientific Investigations, University of Wollongong, garcia@uow.edu.au

Marcio H. F. Bettega

Federal University of Parana

Follow this and additional works at: https://ro.uow.edu.au/eispapers1

Part of the Engineering Commons, and the Science and Technology Studies Commons

Research Online is the open access institutional repository for the University of Wollongong. For further information contact the UOW Library: research-pubs@uow.edu.au 


\title{
Theoretical study on positron scattering by benzene over a broad energy range
}

\author{
Abstract \\ In this paper two theoretical methodologies, the Schwinger multichannel (SMC) method and the \\ independent atom model with the screening corrected additivity rule (IAM-SCAR), were employed to study \\ positron scattering by benzene over a broad impact energy range. The SMC calculations were carried out \\ in the static plus polarization approximation, accounting for the elastic channel, for impact energies up to \\ $20 \mathrm{eV}$. The IAM-SCAR method covered energies up to $1000 \mathrm{eV}$ to provide total, elastic, ionization, \\ excitation, and positronium formation cross sections. In the low-energy region we discuss how the \\ description of the polarization effects affects the cross sections. In particular, our calculations support \\ the existence of a bound state in the positron scattering by benzene, in agreement with previous \\ predictions by Young and Surko [Phys. Rev. A 77, 052704 (2008)NIMBEU0168-583X10.1103/ \\ PhysRevA.77.052704]. \\ Disciplines \\ Engineering | Science and Technology Studies \\ Publication Details \\ Barbosa, A. Souza., Blanco, F., Garcia, G. \& Bettega, M. H. F. (2019). Theoretical study on positron \\ scattering by benzene over a broad energy range. Physical Review A, 100 (4), 042705-1-042705-7.
}




\title{
Theoretical study on positron scattering by benzene over a broad energy range
}

\author{
Alessandra Souza Barbosa $\odot,{ }^{1, *}$ Francisco Blanco, ${ }^{2}$ Gustavo García, ${ }^{3,4}$ and M. H. F. Bettega ${ }^{1}$ \\ ${ }^{1}$ Departamento de Física, Universidade Federal do Paraná, Caixa Postal 19044, 81531-980 Curitiba, Paraná, Brazil \\ ${ }^{2}$ Departamento de Estructura de la Materia, Física Térmica y Electrónica, Universidad Complutense de Madrid, \\ Plaza de Ciencias 1, 28040 Madrid, Spain \\ ${ }^{3}$ Instituto de Física Fundamental, Consejo Superior de Investigaciones Científicas, Serrano 113-bis, 28006 Madrid, Spain \\ ${ }^{4}$ Centre for Medical Radiation Physics, University of Wollongong, NSW 2522, Australia
}

(Received 16 May 2019; published 9 October 2019)

\begin{abstract}
In this paper two theoretical methodologies, the Schwinger multichannel (SMC) method and the independent atom model with the screening corrected additivity rule (IAM-SCAR), were employed to study positron scattering by benzene over a broad impact energy range. The SMC calculations were carried out in the static plus polarization approximation, accounting for the elastic channel, for impact energies up to $20 \mathrm{eV}$. The IAM-SCAR method covered energies up to $1000 \mathrm{eV}$ to provide total, elastic, ionization, excitation, and positronium formation cross sections. In the low-energy region we discuss how the description of the polarization effects affects the cross sections. In particular, our calculations support the existence of a bound state in the positron scattering by benzene, in agreement with previous predictions by Young and Surko [Phys. Rev. A 77, 052704 (2008)].
\end{abstract}

DOI: 10.1103/PhysRevA.100.042705

\section{INTRODUCTION}

Positron scattering by molecules has attracted considerable attention due to the application of positron physics in different areas, such as medicine, astrophysics, and technology. Due to the basic nature of positron interactions with matter, positronscattering studies have become one of the cornerstones that have lead to the understanding of the underlying physics of these applications [1-3]. However, due to some intrinsic difficulties related to the positron physics, the description of the positron-molecule interactions is not an easy task. Even with the advent of better computers and new methodologies, the calculated cross sections still present discrepancies when compared to the experiments. In the low-energy region the difficulties in dealing with this collision problem are mainly related to the angular resolution of the experimental apparatus and to limitation in the description of the positron-molecule interactions by the theoretical methods (e.g., polarization effects and the positronium formation channel).

Benzene is the simplest aromatic hydrocarbon and is a highly symmetric molecule. Although this molecule has received considerable attention in electron-scattering studies, the same cannot be said about positron-scattering studies. Regarding the available data for positron-benzene scattering, we highlight the pioneering study of Sueoka [4], from 1988, which reported total cross sections (TCS) for positron and electron scattering by benzene, with impact energies up to $400 \mathrm{eV}$. In particular, the author reported the dependence of the TCS with the strength of the magnetic field and thus, the TCS were tabulated by considering measurements with different values of the magnetic field adopted for different energy intervals [4]. The author observed that the TCS

\footnotetext{
*alessandra@fisica.ufpr.br
}

presented a sharp increase for energies below $2.5 \mathrm{eV}$ (where the author adopted the value of $3.6 \mathrm{G}$ for the magnetic field). It is also worth mentioning that the reported TCS for positron collisions were not corrected for forward angle scattering effects. Makochekanwa et al. [5] measured TCS for electron and positron scattering by benzene and hexafluorobenzene. The reported TCS for positron scattering by benzene have not displayed the sharp increase at low energies observed by Sueoka [4]. The authors corrected the TCS for the forwardscattering effects but used the differential cross sections (DCS) from electron-benzene collision, since there were no available DCS for positron-benzene collision. Kimura et al. [6] compared the TCS for electron and positron scattering by benzene, focusing on the behavior of the TCS at low energy. The authors discussed the different behaviors of the TCS depending on the incident particle: for electrons the authors observed a sharp increase, which was explained in terms of a virtual state, and for positrons the authors observed a drop in the TCS. These two distinct behaviors were attributed to the balance between the attractive and repulsive interactions in electron and positron molecule collision. Zecca et al. also reported total cross sections for positron scattering by benzene, cyclohexane, and aniline in the energy range from 0.2 to $20 \mathrm{eV}$ [7]. The authors have compared their measured TCS with the results of Sueoka [4] and of Makochekanwa et al. [5] and observed a good agreement with the results of Sueoka [4]. The TCS of Zecca et al. [7] were also not corrected for forward angle scattering effects.

On the other hand, theoretical results for positron-benzene scattering are somewhat scarce, with only two studies reported in the literature. Continuum multiple scattering was employed by Kimura et al. [6] to compute TCS for positron scattering by benzene. The results agree with the experimental TCS measured by the same authors, but disagree with the TCS measured by Sueoka [4] and by Zecca et al. [7]. 
Occhigrossi and Gianturco [8] employed a single-center expansion technique along with a phenomenological (parameter-free) expression to the correlation-polarization potential to compute elastic integral cross sections for a series of hydrocarbons, including benzene. In particular, the calculated integral cross section of Occhigrossi and Gianturco agrees qualitatively well with the TCS of Sueoka [4] and of Zecca et al. [7]. As pointed out by Brunger et al. in their recent review on recommended positron-scattering cross sections for positron-molecule collisions [9], due to the importance of benzene in organic chemistry, there is no doubt on the necessity of further studies on positron scattering by this molecule.

Recently our group has lead some efforts in the study of positron scattering by molecules. Besides working with the biologically relevant molecules pyrimidine [10] and tetrahydrofuran [11], we have also carried out systematic studies in order to investigate the description of the polarization effects for the small nonpolar molecules allene [12] and silane [13]. In particular, only recently, in the calculations for the allene molecule, an $a b$ initio scattering method was able to support a bound state in the positron-molecule scattering [12]. It is worth mentioning that the presence of a virtual or a bound state in the scattering cross section is key for the description of positron annihilation [14] in a molecular environment.

In this paper, a theoretical study on positron scattering by benzene for impact energies up to $1000 \mathrm{eV}$ is presented. The $a b$ initio Schwinger multichannel (SMC) method [15,16] was employed to calculate elastic integral and differential cross sections for impact energies up to $20 \mathrm{eV}$, whereas the independent atom model with the screening corrected additivity rule, including interference (IAM-SCAR+I) effects, was employed to calculate differential, elastic, ionization, excitation, positronium formation, and total cross sections for impact energies up to $1000 \mathrm{eV}$. The low-energy behavior of the cross section is discussed in reference to two calculations carried out with the SMC method, which differ by the description of the polarization effects. We compare our results with the available data from the literature $[4,5,7]$ and we also propose a correction for the experimental results of Zecca et al. [7].

The remainder of this paper is as follows. Both theoretical methods and computational procedures of the calculation are summarized in Sec. II. The results are presented and discussed in Sec. III, and we close the paper in Sec. IV with a brief summary of the present results.

\section{THEORY AND COMPUTATIONAL DETAILS}

\section{A. Schwinger multichannel method}

The elastic cross sections presented here were calculated with the Schwinger multichannel method as implemented for positron-molecule scattering. Since the method has been described in detail in several publications $[15,16]$, here we will only discuss those aspects of the SMC method that are relevant to the present calculations.

All calculations were carried out in the optimized geometry of benzene (shown in Fig. 1), obtained previously in our work on electron scattering by the same molecule [18]. The $\mathrm{DZV}++(2 d, 1 p)$ basis set as implemented in the package GAMESS [19] was used in the bound-state and scattering

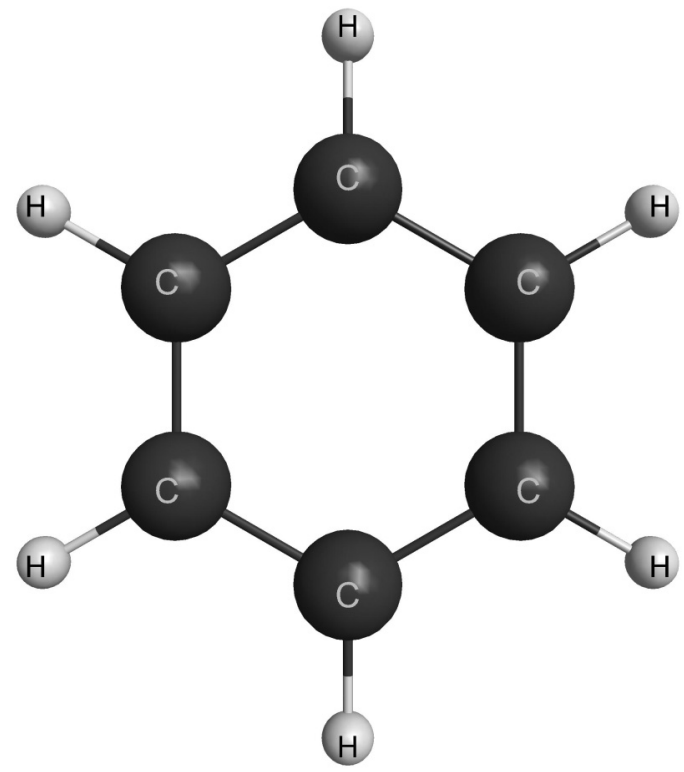

FIG. 1. Geometrical structure of benzene (generated with MACMOLPLT) [17].

calculations. Finally, although benzene belongs to the $D_{6 h}$ symmetry group, our scattering calculations were carried out in the $D_{2 h}$ symmetry group, since the SMC code only deals with Abelian symmetry groups. It should be noted that if the same polarization criterion is employed (the same number of hole, particle, and scattering orbitals) the ICS and the DCS are not affected by the symmetry group used in the calculations. In addition, most of the positron-molecule physics in the low-energy region is discussed in view of the $s$-wave cross section, which belongs to the totally irreducible representation regardless of which symmetry group is employed in the calculations.

Our SMC calculations were carried out in the static plus polarization (SP) approximation, where the direct space is composed by configuration state functions (CSFs) of the form

$$
\left|\chi_{j}\right\rangle=\left|\Phi_{1}\right\rangle \otimes\left|\varphi_{j}\right\rangle \oplus\left|\Phi_{i}\right\rangle \otimes\left|\varphi_{j}\right\rangle
$$

where $\left|\Phi_{1}\right\rangle$ represents the ground state of the molecule obtained at the Hartree-Fock level and $\left|\varphi_{j}\right\rangle$ is a single-particle orbital used to expand the positron scattering orbital (see below) and $\left|\Phi_{i}\right\rangle$ is obtained from virtual single excitations of the target from the hole (occupied) orbitals to a set of particle (unoccupied) orbitals. In the present calculations we considered the 15 outermost occupied orbitals as hole orbitals whereas the particle orbitals were represented by a set of modified virtual orbitals (MVOs) [20], obtained through the diagonalization of the Fock operator with charge +8 . These MVOs, along with the 21 occupied orbitals, were used as scattering orbitals. We carried out two calculations labeled as SP-1 and SP-2 that differ on the number of MVOs employed. In the SP-1 calculations the first 50 MVOs were employed, whereas in the SP-2 calculations the first 75 MVOs were employed, for a total of 53424 CSFs and 108174 CSFs, respectively. In Table I we summarize the number of CSFs employed in each irreducible representation of the $D_{2 h}$ symmetry group. 
TABLE I. Details of the calculations carried out employing the SMC method: number of configuration state functions in each irreducible representation (IR) of the $D_{2 h}$ symmetry group.

\begin{tabular}{lcc}
\hline \hline IR & SP-1 $(15 h 50 p 71 s)$ & SP-2 $(15 h 75 p 96 s)$ \\
\hline$A_{g}$ & 7227 & 15425 \\
$A_{u}$ & 6134 & 11628 \\
$B_{1 g}$ & 7061 & 15203 \\
$B_{1 u}$ & 6287 & 11830 \\
$B_{2 g}$ & 6175 & 11657 \\
$B_{2 u}$ & 7186 & 15391 \\
$B_{3 g}$ & 6250 & 11805 \\
$B_{3 u}$ & 7104 & 15235 \\
\hline
\end{tabular}

\section{B. Independent atom model with screening corrected additivity rule}

The screening corrected additivity rule, within the framework of the independent atom model (IAM-SCAR), as used in this paper has been described in previous publications $[21,22]$. It has been successfully applied in preceding studies for several biologically relevant molecules (see Ref. [23] and references therein), typically in the range of 0.1 - to $10000-\mathrm{eV}$ incident energy.

IAM-SCAR basically relies on the optical potential method [24], initially applied to the constituent atoms of the molecule, i.e., $\mathrm{C}$ and $\mathrm{H}$. The atomic scattering potential is then represented by

$$
V(r)=V_{s}(r)+V_{p}(r)-i V_{a}(r) .
$$

The real part of Eq. (2) drives the elastic-scattering dynamics and includes the electrostatic $\left[V_{s}(r)\right]$ and polarization $\left[V_{p}(r)\right]$ interactions. The imaginary part $\left[V_{a}(r)\right]$ describes all inelastic processes that are considered as absorptions from the incident positron beam. Owing to this last term in Eq. (2), the optical model potential method yields a complex phase shift $\delta_{l}=$ $\lambda_{l}+i \mu_{l}$. This allows for the calculation of the atomic scattering amplitudes, from which the corresponding differential and integral elastic cross sections as well as the integral inelastic and therefore the total cross sections are derived.

The static potential was obtained from the charge density derived from Hartree-Fock atomic wave functions, using a procedure analogous to that of Reid and Wadehra [25]. The dipole plus quadrupole polarization potential was developed from that reported by McEachran et al. [26] for $\mathrm{Ne}$, but scaled by constants in order to match the known dipole and quadrupole polarizabilities of the $\mathrm{C}$ and $\mathrm{H}$ atoms (see Ref. [21] for details). The absorption potential accounts for the electronic excitations, positronium formation, and direct ionization. However, owing to the challenging nature of representing the Ps formation channel, the definition of the threshold energy for the absorption potential can be critical. Our recent improvements to the treatment of Ps formation were outlined in detail previously $[22,23]$. In brief we maintain the energy dependent threshold $\Delta(E)$ defined in Ref. [21], by necessity coinciding with the well-known Ps formation threshold of $\Delta_{p}=I-6.8 \mathrm{eV}$ (where $I=$ ionization threshold), for lower energies and the lowest optically allowed excitation transition $\Delta$ for higher impact energies but applying the smooth tran- sition in threshold energy from low to high impact energy as proposed in Ref. [23]:

$$
\Delta(E)=\Delta-\frac{\left(\Delta-\Delta_{p}\right)}{\left[1+\left(\frac{E}{3 I}-1\right)^{2}\right]} .
$$

Once we have calculated the atomic scattering amplitudes, the IAM-SCAR procedure [27] gives the molecular scattering amplitudes, $F(\theta)$, from those of the constituent atoms, $f_{i}(\theta)$, according to the expression

$$
F(\theta)=\sum_{\text {atoms }} f_{i}(\theta) e^{i \vec{q} \cdot \vec{r}_{i}},
$$

where the momentum transfer is $\vec{q}=\vec{k}_{f}-\vec{k}_{i}$ and the atomic positions are given by $\vec{r}_{i}$. In this calculation we incorporate the recent improvement of considering interference effects [28]. This updated version is known as IAM-SCAR+I and basically provides the molecular differential cross section $\left(d \sigma_{\text {molecule }}^{\text {elastic }} / d \omega\right)$ as a combination of the multicenter atomic amplitudes given by

$$
\begin{aligned}
\frac{d \sigma_{\text {molecule }}^{\text {elastic }}}{d \Omega} & =\sum_{i j} f_{i}(\theta) f_{j}^{*}(\theta) \frac{\sin q r_{i j}}{q r_{i j}} \\
& =\sum_{i}\left|f_{i}(\theta)\right|^{2}+\sum_{i \neq j} f_{i}(\theta) f_{j}^{*}(\theta) \frac{\sin q r_{i j}}{q r_{i j}} .
\end{aligned}
$$

Here the interference term is the second summation in Eq. (5). In this case $q \equiv|\vec{q}|=2 k \sin \left(\frac{\theta}{2}\right)$ is the momentum transfer and $r_{i j}$ is the distance between atoms $i$ and $j$.

By integrating Eq. (5), the corresponding molecular integral cross sections are represented by

$$
\sigma_{\text {molecule }}^{\text {total }}=\sum_{\text {atoms }} s_{i} \sigma_{\text {atom }_{i}}^{\text {total }}+\sigma^{\text {interference }} .
$$

The factor $s_{i}$ is a screening correction, reducing the contribution of each atom to the total molecular cross section $\left(0 \leqslant s_{i} \leqslant 1\right)$ based on the position of the atom within the molecule. This accounts for the fact that, as the energy of the incoming particle decreases, the atomic cross sections overlap, requiring a reduction of their relative contribution to the summation [29]. The remaining term in Eq. (6) is determined from

$$
\sigma_{\text {interference }} \equiv \int d \Omega \sum_{i \neq j} f_{i}(\theta) f_{j}^{*}(\theta) \frac{\sin q r_{i j}}{q r_{i j}} .
$$

Including interference terms in the calculation of both integral and differential cross sections for molecular targets eliminates the inconsistency between the differential and integral cross-section values, which is inherent to the IAM-SCAR method [29]. This means that no additional normalization procedure is required by the IAM-SCAR $+\mathrm{I}$ approach in order to fulfill the optical theorem [29].

\section{RESULTS}

In Fig. 2 we present the calculated cross sections for positron scattering by benzene employing the SMC method for energies up to $20 \mathrm{eV}$ and the IAM-SCAR+I method for energies up to $1000 \mathrm{eV}$ and compare with available results 


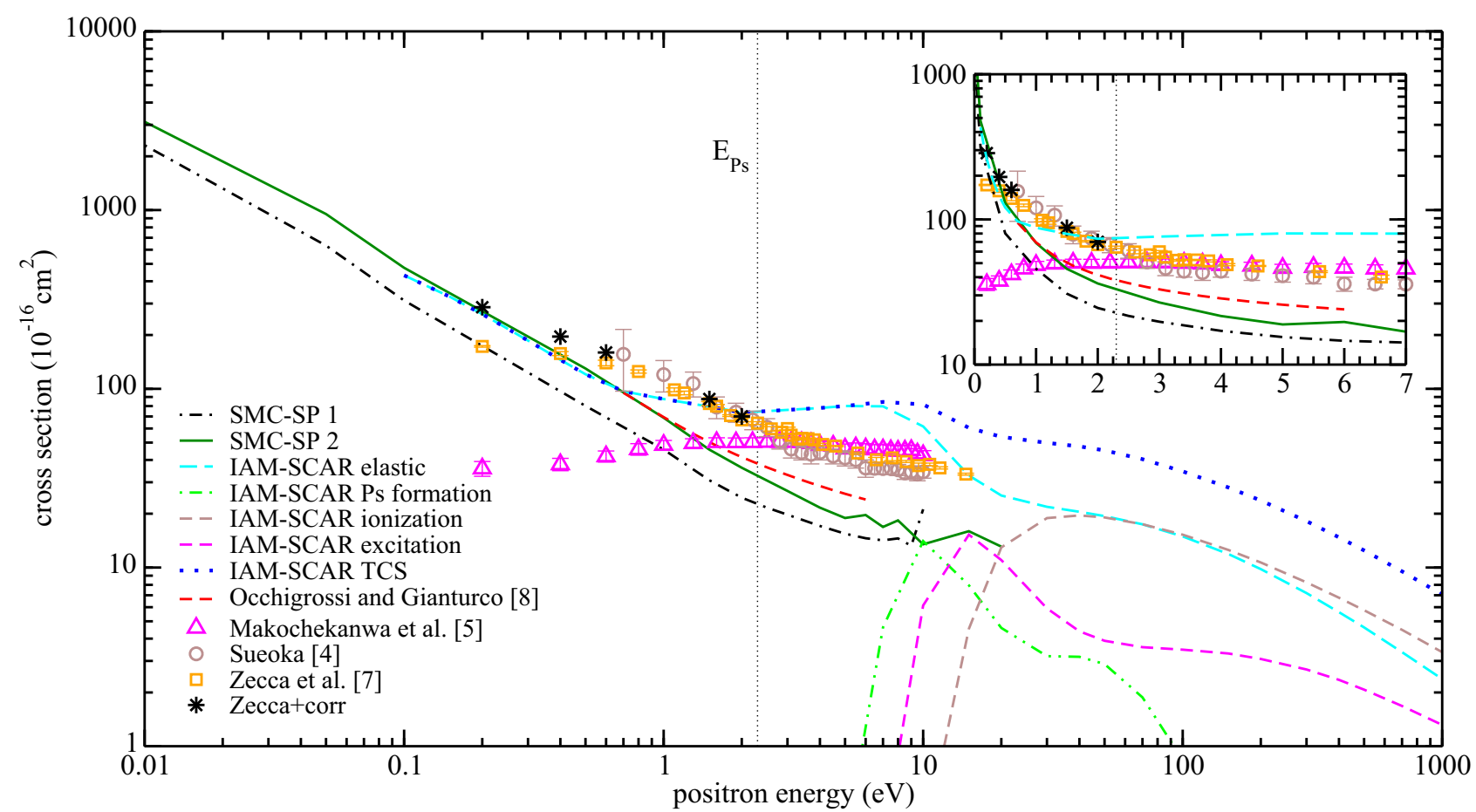

FIG. 2. Present positron-benzene calculated cross sections (SMC and IAM-SCAR results) obtained over a broad energy range, compared with available data from literature. The vertical dotted line indicates the positronium formation threshold, which was estimated at $2.3 \mathrm{eV}$. In the inset, the same data are presented but in a reduced energy scale.

from the literature. The elastic cross sections obtained with the SMC method were calculated in the static plus polarization approximation employing different numbers of MVOs as particle and scattering orbitals and, consequently, different number of configurations. These calculations are labeled as SP-1 and SP-2, where the SP-1 calculations employed 53424 configurations and the SP-2 included 108174 configurations. For purpose of comparison, the experimental total cross sections reported by Makochekanwa [5], Sueoka [4], and Zecca et al. [7] and the theoretical results of Occhigrossi and Gianturco [8] are also shown in this figure. In the inset of Fig. 2, the same results are presented but in a reduced energy scale. Also presented in this figure is the proposed correction for the experimental data of Zecca et al. [7], which will be further discussed in the next paragraphs.

From Fig. 2 it is seen that the improvement in the description of the polarization effects in the SMC calculations, from SP-1 to SP-2, increases the cross sections, especially in the low-energy region. When comparing the elastic cross sections obtained with both methods, the IAM-SCAR+I and the SMC-SP 2, there is an overall good agreement, particularly for energies lower than $1 \mathrm{eV}$. This indicates the appropriate description of the polarization effects, which are dominant at these low energies, given by both calculation methods. As the impact energy increases, the calculated IAM-SCAR+I elastic cross sections increase whereas the SMC calculated cross sections decrease. Also presented in this figure are our calculated cross sections for the positronium (Ps) formation, electronic excitation, and ionization, obtained with the IAM$\mathrm{SCAR}+\mathrm{I}$ method. Since molecular features are not considered by the IAM-SCAR+I procedure we do not expect to be reliable in the range $1-10 \mathrm{eV}$ where most of the inelastic channels are opening. To the best of our knowledge, despite the importance of these channels at higher impact energies, there are no experimental or theoretical results for the positronium formation, electronic excitation, and ionization channels available in the literature. When comparing the calculated elastic cross sections with the experimental TCS, one can observe a fair agreement between all theories and the experimental data from Sueoka [4] and from Zecca et al. [7]. This agreement is seen regarding the fact that, at lower energies, the experimental cross section suffers with the apparatus's angular resolution being, usually, underestimated [9]. The poor angular resolution of the apparatus used by Makochekanwa et al. [5] is the reason for the dramatic decrease of the TCS as the impact energies decrease.

The angular resolution of the apparatus is related with experimental difficulty to properly discriminate the scattered positrons in the forward region. Positrons scattered at angles lower than a specific angle $\theta_{m}$ are considered as nonscattered positrons and, consequently, the experimental TCS are underestimated of their true value [9]. This issue has been very well described previously by Karwasz et al. [30] for the benzene molecule. Following a similar procedure we estimated a correction for the TCS $\sigma_{\text {corr }}$ given by

$$
\sigma_{\text {corr }}=2 \pi \int_{0}^{\theta_{m}}\left(\frac{d \sigma}{d \Omega}\right)(\theta) \sin \theta d \theta,
$$

where $d \sigma / d \Omega$ is the differential cross sections obtained with the SMC method. The above equation includes only the forward angle region in the integration. Thereby, with the aim of estimating the influence of the low angle region in the total cross sections and to propose a correction to the 

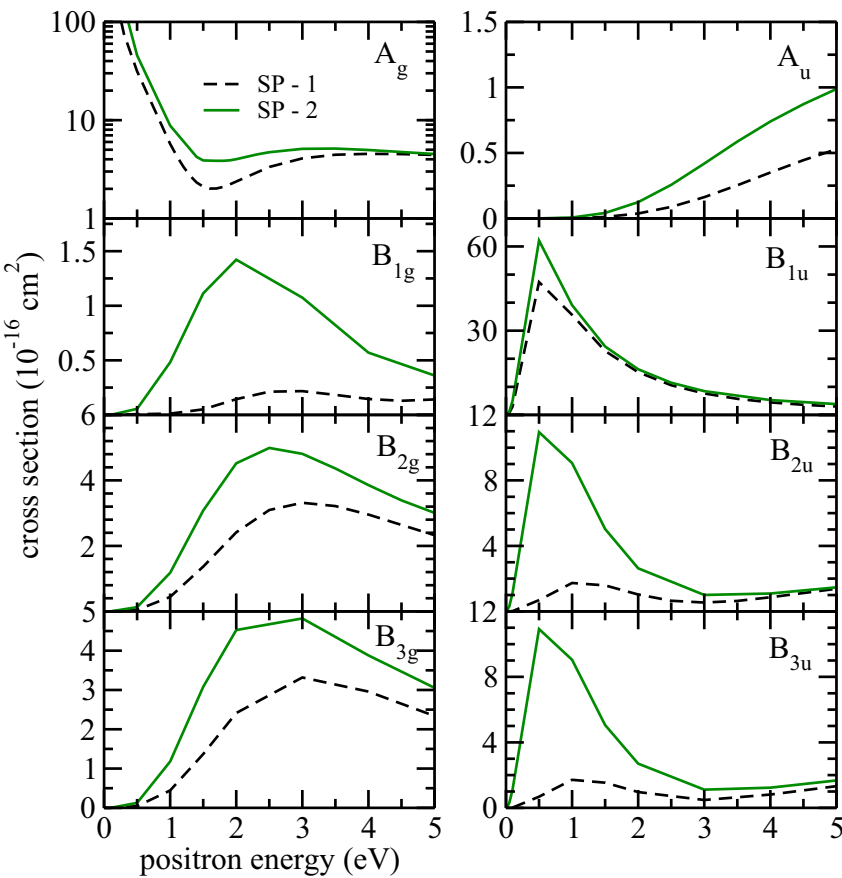

FIG. 3. Symmetry decomposition, according to the $D_{2 h}$ symmetry group, of the calculated ICS obtained with the SMC method. See text for discussion.

experimental data of Zecca et al. [7], we employed our calculated SMC-SP 2 DCSs and the available resolution of the experimental apparatus [9] $\left(\theta_{s}\right)$ to calculate $\sigma_{\text {corr }}$, which was added to the experimental data at selected energies. These results are shown in Fig. 2 and are labeled as Zecca+corr.

The symmetry decomposition of the SMC calculated cross sections, according to the $\mathrm{D}_{2 h}$ symmetry group, for impact energies up to $5 \mathrm{eV}$, is presented in Fig. 3. From this figure it is seen that the low-energy behavior of the ICS, which dramatically increases, is predominantly due to the totally symmetric irreducible representation $A_{g}$. This will be further discussed in the next paragraph with the aid of the $s$-wave cross section and eigenphase. Additionally, in Fig. 3 the improvement in the cross sections is also remarkable, as the polarization effects are better described not only in the $A_{g}$ but also in the other irreducible representations.

In Fig. 4 we present the $s$-wave cross section and the respective eigenphase for both calculations, SP-1 and SP-2, carried out with the SMC method. Here two features seen in the cross section and in the eigenphase must be addressed: the minimum in the cross section, where the corresponding eigenphase crosses zero, and the low-energy behavior of the cross section, which increases as the impact energy goes toward zero, also corresponding to an increase in the corresponding eigenphase. The first feature is explained by the net potential felt by the incoming positron, which is the combination of the attractive polarization potential and the repulsive static potential. The energy where the $s$-wave eigenphase crosses zero and changes sign (from negative to positive as the energy increases) corresponds to the change in the net potential (from attractive to repulsive) felt by the incoming positron.

The second feature is the dramatic increase in the $s$-wave cross section, as seen in Fig. 4, which can be explained in

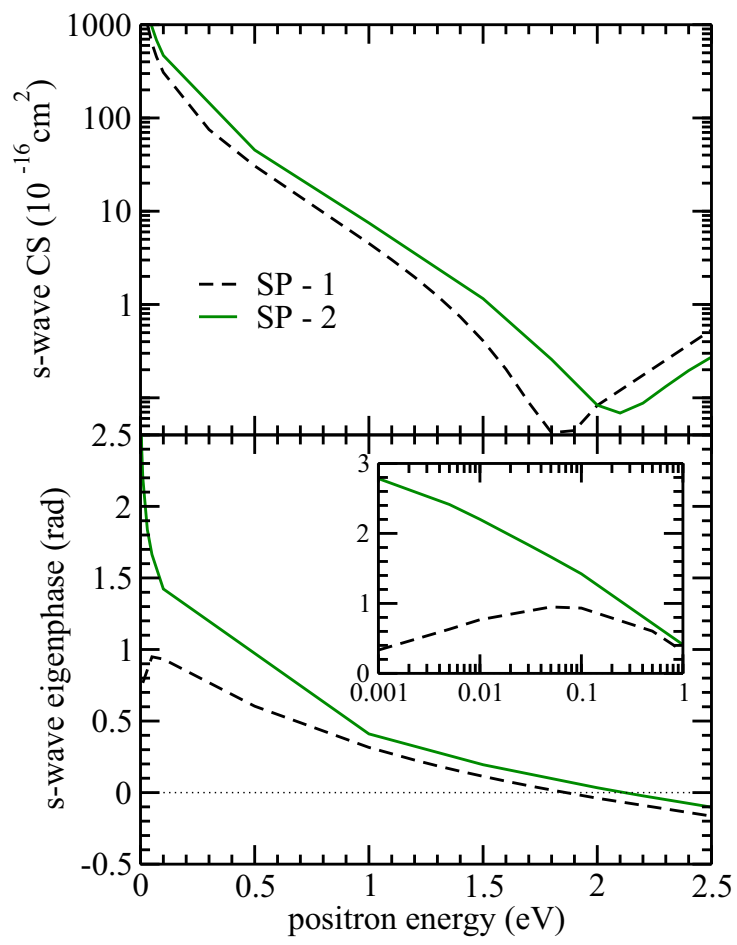

FIG. 4. SMC calculated $s$-wave cross section (upper panel) and eigenphase (lower panel). The inset in the lower panel also presents the $s$-wave eigenphase but employing a more suitable log scale at the impact energy axis.

terms of a virtual or a bound state, and, from the analysis of the $s$-wave eigenphase, it is possible to determine which one takes place. Ideally, if a virtual state takes place, the $s$-wave eigenphase goes to $\pi / 2$ as the impact energy goes towards zero, while in the case of a bound state the $s$-wave eigenphase goes towards $\pi$ [31]. In real systems when a virtual state takes place, the $s$-wave eigenphase goes towards $\pi / 2$ and then dramatically fall down to zero. This is the behavior presented by the SP-1 eigenphase, indicating that this calculation supports a virtual state in the positron-benzene scattering. As for the SP-2 calculation, one can see that the $s$-wave eigenphase increases, going towards $\pi$, which means that the potential supports a bound state. The scattering length (SL) also provides an indication of the presence of a virtual or a bound state. The SL can be calculated as

$$
\mathrm{SL}=-\lim _{k \rightarrow 0} \frac{1}{k} \tan \delta_{0}(k),
$$

where the $\delta_{0}$ is the $s$-wave eigenphase and $k$ is the momentum of the incoming projectile [31]. Usually, the presence of a virtual state corresponds to a negative value of SL, while if the potential supports a bound state the SL is positive. Employing our calculated $s$-wave eigenphase to estimate the SL we obtained SL $=+42.8 a_{0}$ for the SP- 2 calculation, corroborating the presence of a bound state. This result is in agreement with previous experimental data that predicts a bound state in the positron-benzene interactions [32].

In Fig. 5 our calculated differential cross sections are presented at energies up to $20 \mathrm{eV}$, obtained with both methods and at higher energies obtained only with the IAM-SCAR+I 

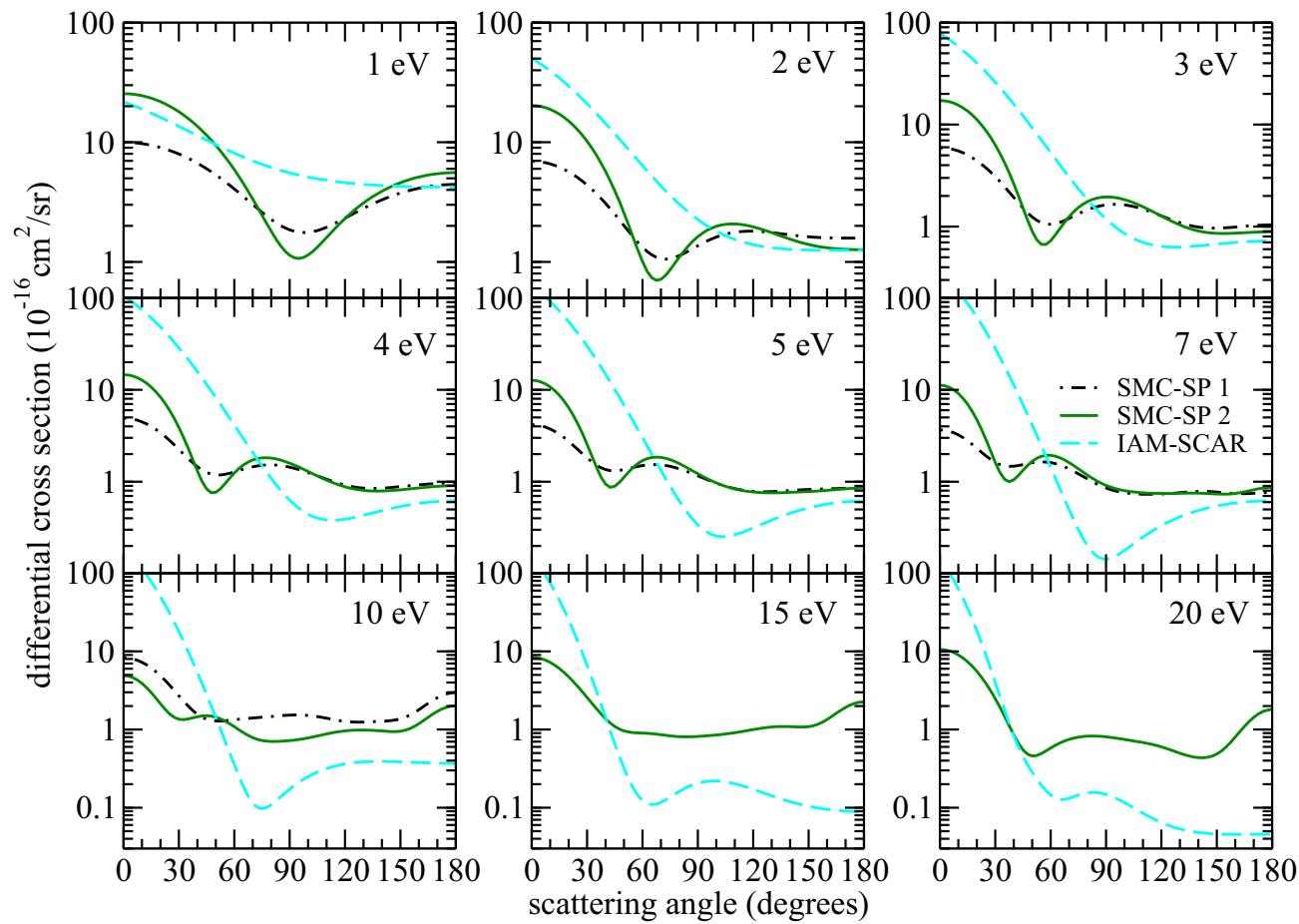

FIG. 5. Comparison of the present calculated differential cross section for positron scattering by benzene, obtained with SMC and IAM-SCAR methods. See text for discussion.

method. Regarding the comparison of both SMC calculations, in Fig. 5, we found that as the polarization effects are better described (from SP-1 to SP-2 calculation) the forward angle region is improved. This strengthens the necessity of new theoretical studies that aim to discuss and improve the description of the polarization effects in positron-scattering calculations.

Apart from the low-energy positron scattering features an additional interest of the present calculation is to provide positron scattering cross sections for high incident energies up to $1000 \mathrm{eV}$. Modelers customarily use electron scattering cross sections instead of those for positrons (generally more

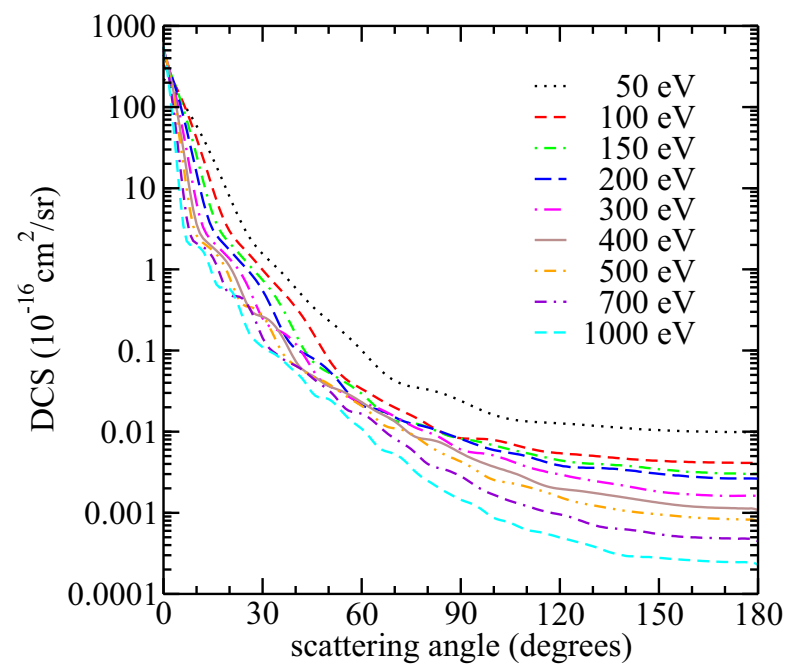

FIG. 6. Present positron-benzene calculated differential cross section at higher energies, obtained with the IAM-SCAR method. scarce) by assuming they are coincident for high energies. The present paper gives the opportunity of using both integral and differential positron scattering cross sections for modeling positron transport up to $1000 \mathrm{eV}$. In particular, it should be noted that the DCSs shown in Fig. 6, for impact energies from 50 to $1000 \mathrm{eV}$, provide by simple integration the momentum transfer cross sections

$$
\sigma_{\mathrm{MT}}=2 \pi \int_{0}^{\pi}(1-\cos \theta)(d \sigma / d \Omega)(\theta) \sin \theta d \theta
$$

which are relevant data for transport equations.

\section{SUMMARY}

Here an extensive work on theoretical description of positron scattering by benzene molecules was presented. Employing two different methodologies, the SMC and the IAM-SCAR methods, we were able to provide elastic integral, elastic differential, total, positronium formation, ionization, and excitation cross sections calculated for positron impact energies over a broad range (from 0.001 to $1000 \mathrm{eV}$ ). We compared our calculated integral elastic and total cross sections with available experimental TCSs from the literature and found, in general, a fair agreement. We also presented ionization, positronium formation, and excitation cross sections, calculated with the IAM-SCAR method, and to the best of our knowledge no other theoretical or experimental results for these channels are available in the literature, indicating the urgency for new studies on positron-benzene interactions.

With the SMC method we discussed the description of the polarization effects by performing two different calculations, which employed different configuration state function spaces. 
In particular, it is remarkable that as the polarization effects description is improved our scattering calculations are able to predict the existence of a bound state in the positron-benzene interactions. This issue was supported by analysis of the $s$-wave cross sections and eigenphase.

Finally, we also presented differential cross sections for impact energies from 1 to $20 \mathrm{eV}$ obtained with both methods and for impact energies up to $1000 \mathrm{eV}$ obtained by the IAMSCAR + I method. The DCSs obtained with the SMC method were employed to estimate a correction for the experimental TCSs of Zecca et al. [7] due to the forward angular resolution effects.

\section{ACKNOWLEDGMENTS}

A.S.B. acknowledges support from Brazilian Agency Coordenação de Aperfeiçoamento de Pessoal de Nível Superior. M.H.F.B. acknowledges support from the Brazilian agency Conselho Nacional de Desenvolvimento Científico e Tecnológico and from Financiadora de Estudos e Projetos (under project CTInfra). A.S.B. and M.H.F.B. also acknowledge computational support from Prof. Carlos M. de Carvalho at LFTC-DFis-UFPR and at LCPAD-UFPR, and from CENAPAD-SP. F.B. and G.G. acknowledge partial financial support from the Spanish Ministerio de Ciencia, Innovación y Universidades (Project No. FIS2016-80440).
[1] C. M. Surko, G. F. Gribakin, and S. J. Buckman, J. Phys. B 38, R57 (2005).

[2] L. Chiari and A. Zecca, Eur. Phys. J. D 68, 297 (2014).

[3] J. R. Danielson, D. H. E. Dubin, R. G. Greaves, and C. M. Surko, Rev. Mod. Phys. 87, 247 (2015).

[4] O. Sueoka, J. Phys. B 21, L631 (1988).

[5] C. Makochekanwa, O. Sueoka, and M. Kimura, Phys. Rev. A 68, 032707 (2003).

[6] M. Kimura, C. Makochekanwa, and O. Sueoka, J. Phys. B 37, 1461 (2004).

[7] A. Zecca, N. Moser, C. Perazzolli, A. Salemi, and M. J. Brunger, Phys. Rev. A 76, 022708 (2007).

[8] A. Occhigrossi and F. A. Gianturco, J. Phys. B 36, 1383 (2003).

[9] M. J. Brunger, S. J. Buckman, and K. Ratnavelu, J. Phys. Chem. Ref. Data 46, 023102 (2017).

[10] A. S. Barbosa, D. F. Pastega, and M. H. F. Bettega, J. Chem. Phys. 143, 244316 (2015).

[11] A. S. Barbosa and M. H. F. Bettega, J. Chem. Phys. 150, 184305 (2019).

[12] A. S. Barbosa, S. A. Sanchez, and M. H. F. Bettega, Phys. Rev. A 96, 062706 (2017).

[13] A. S. Barbosa and M. H. F. Bettega, Phys. Rev. A 96, 042715 (2017).

[14] G. F. Gribakin, J. A. Young, and C. M. Surko, Rev. Mod. Phys. 82, 2557 (2010).

[15] J. S. E. Germano and M. A. P. Lima, Phys. Rev. A 47, 3976 (1993).

[16] E. P. da Silva, J. S. E. Germano, and M. A. P. Lima, Phys. Rev. A 49, R1527 (1994).

[17] B. M. Bode and M. S. Gordon, J. Mol. Graphics Mod. 16, 133 (1998).
[18] A. S. Barbosa and M. H. F. Bettega, J. Chem. Phys. 146, 154302 (2017).

[19] M. W. Schmidt, K. K. Baldridge, J. A. Boatz, S. T. Elbert, M. S. Gordon, J. H. Jensen, S. Koseki, N. Matsunaga, K. A. Nguyen, S. J. Su, T. L. Windus, M. Dupuis, and J. A. Montgomery, J. Comput. Chem. 14, 1347 (1993).

[20] C. W. Bauschlicher, J. Chem. Phys. 72, 880 (1980).

[21] L. Chiari, A. Zecca, S. Girardi, E. Trainotti, G. García, F. Blanco, R. P. McEachran, and M. J. Brunger, J. Phys. B 45, 215206 (2012).

[22] F. Blanco, A. Roldan, K. Krupa, R. P. McEachran, R. D. White, S. Marjanovic, Z. L. Petrovic, M. J. Brunger, J. R. Machacek, S. J. Buckman, J. P. Sullivan, L. Chiari, P. Limão-Vieira, and G. García, J. Phys. B 49, 145001 (2016).

[23] D. Stevens, T. J. Babij, J. R. Machacek, S. J. Buckman, M. J. Brunger, R. D. White, G. García, F. Blanco, L. Ellis-Gibbings, and J. P. Sullivan, J. Chem. Phys. 148, 144308 (2018).

[24] F. Blanco and G. García, Phys. Rev. A 67, 022701 (2003).

[25] D. D. Reid and J. M. Wadehra, J. Phys. B 29, L127 (1996).

[26] R. P. McEachran, A. G. Ryman, A. D. Stauffer, and D. L. Morgan, J. Phys. B 10, 663 (1977).

[27] L. Chiari, P. Palihawadana, J. R. Machacek, C. Makochekanwa, G. García, F. Blanco, R. P. McEachran, M. J. Brunger, S. J. Buckman, and J. P. Sulivan, J. Chem. Phys. 138, 074302 (2013).

[28] F. Blanco, L. Ellis-Gibbings, and G. García, Chem. Phys. Lett. 645, 71 (2016).

[29] F. Blanco and G. García, Phys. Lett. A 317, 458 (2003).

[30] G. Karwasz, A. Karbowski, Z. Idziaszek, and R. S. Brusa, Nucl. Instrum. Methods B 266, 471 (2008).

[31] C. J. Joachain, Quantum Collision Theory (North-Holland, Amsterdam, 1975).

[32] J. A. Young and C. M. Surko, Phys. Rev. A 77, 052704 (2008). 